\section{Galileo's observation of Neptune}

KOWAL AND DRAKE ${ }^{1}$ have found a record in Galileo's notebooks of Neptune's position-the most precise placing being that of 28 January 1613 (Besselian date 1613.08). They note, however, a disagreement of 1 arc min between the position observed $(\mathrm{O})$ by Galileo and that predicted by modern calculation (C) - using the orbit of the Jet Propulsion Laboratory, Development Ephemeris no. 102, for the C. They suggest that the effect of a yet-undiscovered exterior planet may explain this discrepancy. The 1613 residuals are listed in Table 1. To evaluate conservatively

was made during his search for Jupiter satellites, so Jupiter was in his field of view. The account and drawing for his 28 January 1613 entry both note that Jupiter, star ' $a$ ' (SAO119234), and star 'b' (the planet Neptune) were in a straight line. Kowal and Drake note that the suspect modern ephemeris correctly predicts the same lineup.

This line cuts the ecliptic at a descending angle of $25.9^{\circ}$. (See Table 1 or Fig. 5 of ref. 1.) Thus, if the modern orbit is approximately correct and Galileo accidentally distorted his drawing's scale, then we would expect a false latitude residual $F \Delta \beta$ corresponding to the false longitude residual $F \Delta \lambda$ according to the relation:

$$
F \Delta \beta / F \Delta \lambda=\tan \left(-25.9^{\circ}\right)
$$

Table 1 Residuals (O-C) for 28 January 1613

\begin{tabular}{lllllll}
\hline Geocentric & RA & $\Delta \alpha$ & -42 arcs & Dec & $\Delta \delta$ & +47 arcs \\
Geocentric & long & $\Delta \lambda$ & -57 arcs & lat & $\Delta \beta$ & +26 arcs \\
Heliocentric & long & $\Delta l$ & -56 arcs & lat & $\Delta b$ & +26 arcs \\
\hline
\end{tabular}

the exterior-planet hypothesis, I have computed the strength of the 1613.08 perturbation on Neptune, caused by hypothetical tenth planets in those circular orbits ${ }^{2,3}$ permitted by modern residuals (see Table 2). The elements and masses used were those indicated by least-squares analyse ${ }^{2,3}$ of Neptune longitude residuals, post-discovery as well as M. Lalande's 8 and 10 May 1795 observations (discord, -7 arc s both nights) ${ }^{4}$.

Table 2 shows that: the magnitude of the disturbances produced by the hypothetical exterior planets is at best in the range 10-20 arc $s$ which, even allowing for some mass uncertainty, disagrees with the 1613 residual being nearly 1 arc min; (2) the direction is negative in all the cases listed which agrees with Table 1.

The unlikelihood of a residual as large as 1 arc min is also supported by another line of reasoning. Galileo's observation
Setting $F \Delta \lambda$ equal to the geocentric longitude residual $\Delta \lambda$ of Table 1 , we substitute this into equation (1) and find that the accidental scale-distortion hypothesis predicts a false longitude residual

$$
F \Delta \beta=+28 \text { arc s }
$$

This is within $2 \operatorname{arcs}$ of the observed geocentric latitude residual $\Delta \beta$ of +26 arcs (Table 1).

Kowal and Drake note that our present uncertainty about the motion of Neptune is due to the relative shortness of the planet's continuously observed arc (less than a single full revolution since the 1846 discovery). In other words, a sizeable error in the baseline of the planet's motion may be masked by an alien perturbation. (The baseline error is $A+B t$ in equation (1) of ref. 3. $A$ is the error in longitude at epoch, and $B$ is the error in the mean motion.)

Table 2 Perturbations from hypothetical planets

\begin{tabular}{ccccccc}
\hline & \multicolumn{2}{c}{$\begin{array}{c}\text { Disturber's } \\
\text { mean } \\
\begin{array}{c}\text { Disturber's } \\
\text { distance }\end{array}\end{array}$ long $^{\text {laU) }}$} & $1973.0^{2}$ & 1981.0 & $\begin{array}{c}\text { Disturber's } \\
\text { mass } \\
\left(\boldsymbol{R}_{\odot} / 206265\right)\end{array}$ & \multicolumn{2}{c}{$\begin{array}{c}\text { Longitude perturbations } \\
\text { (arc s) }\end{array}$} \\
75 & $300^{\circ}$ & $304^{\circ}$ & 3.95 & -8.7 & -3.4 & +1.1 \\
& $310^{\circ}$ & $314^{\circ}$ & 4.80 & -11.4 & -5.6 & +1.0 \\
& $320^{\circ}$ & $324^{\circ}$ & 4.11 & -9.5 & -5.6 & +0.6 \\
60 & $310^{\circ}$ & $316^{\circ}$ & 1.55 & -8.4 & -1.6 & +0.8 \\
& $320^{\circ}$ & $326^{\circ}$ & 2.90 & -18.5 & -4.9 & +1.1 \\
& $330^{\circ}$ & $336^{\circ}$ & 2.61 & -18.5 & -6.2 & +0.7 \\
& $340^{\circ}$ & $346^{\circ}$ & 1.98 & -13.8 & -5.7 & +0.3 \\
& $340^{\circ}$ & $348^{\circ}$ & 2.32 & -4.1 & -5.7 & +1.1 \\
& $350^{\circ}$ & $358^{\circ}$ & 1.74 & -10.9 & -6.3 & +0.5 \\
& $0^{\circ}$ & $8^{\circ}$ & 1.24 & -12.3 & -5.7 & +0.1 \\
& $10^{\circ}$ & $18^{\circ}$ & 0.95 & -11.6 & -5.1 & -0.1 \\
\hline
\end{tabular}

However, there is no such thing as a baseline error in latitude motion (no linear expression appears in equation (2) of ref. 3 , compare with equation (1) of ref. 3 ). Thus, if the 1613.08 latitude residuals produced by the hypothetical disturbers of refs 2, 3 are computed, it turns out that they are merely of order of magnitude 1 arc s-a far cry from the observed residual of 26 arcs.

Thus Galileo's illustration of Star SAO119234 and Neptune probably has an erroneous scale. However, if Galileo was at least correct in picturing Neptune closer to the star than modern theory predicts, then a negative 10-20 arc s longitude residual would be consistent with perturbation by an outer planet itself reasonably consistent with later longitude residuals. Hence, the slight nonlinearity of Neptune, star, and Jupiter might not be recognized.

2636 Grand Avenue, San Diego, California, USA

1. Kowal, C. \& Drake, S. Nature 287, 311-313 (1980)

2. Rawlins, D. \& Hammerton, M. Nature 240, 457 (1972). 3. Rawlins, D. \& Hammerton, M. Mon. Not. R. astr. Soc. 162, 261 (1973).

4. Rawlins, D. Astr. J. 75, 856 (1970).

KOWAL AND DRAKE ${ }^{1}$ established that Galileo observed the planet Neptune and actually noted its motion. However, I must comment on their statement that "we are left, then, with the strong possibility that the ephemeris of Neptune is in error by a significant amount...".

It would be virtually impossible for the ephemeris of Neptune to be in error by over 26 arc $s$ in latitude (the component of the residual perpendicular to Neptune's orbital plane) without "the existence of an unknown perturbation". As good fortune would have it, modern observations (since 1910) cover just that part of the sky in which Galileo made his observation (the exact crossing of $\alpha=12 \mathrm{~h}$ occurred in 1943). As such, the orbital plane of Neptune, especially in that part of the sky, is established to within a small fraction of 1 arc s.

We recently attempted to incorporate Galileo's observation into the JPL Planetary Ephemeris. Even with the unrealistically high observation weight of 1 arcs, the attempt proved to be entirely unsuccessful. It was possible to adjust the longitude somewhat, but the 26 arc s latitude residual remained.

On the other hand, a perturbation of $26 \operatorname{arcs}$ in latitude was easily produced using numerical experiments with an additional planet of a few Earth masses, even when the closest approach to Neptune was as distant as $10 \mathrm{AU}$. However, such perturbations usually tend 